\title{
NONLINEAR BEAM DYNAMICS EXPERIMENTS AT THE SRRC
}

\author{
C.C. Kuo ${ }^{\dagger}$, H.P. Chang, J. Chen, K.T. Hsu, K.H. Hu, K.K. Lin, Y.C. Liu, H.J. Tsai, T.S. Ueng \\ Synchrotron Radiation Research Center \\ No.1 R\&D Rd VI, Hsinchu Science-Based Industrial Park, Hsinchu, Taiwan
}

\begin{abstract}
Nonlinear beam dynamics experiments were conducted at the Taiwan Light Source (TLS) of the Synchrotron Radiation Research Center (SRRC). We employed a turn-by-turn BPM system to obtain nonlinear dynamics of the excited bunched beam. Extracted nonlinear parameters are compared with the simulation results.
\end{abstract}

\section{INTRODUCTION}

Nonlinear field is of importance for the design and operation of circular machines and nonlinear beam dynamics has been intensively investigated both theoretically and experimentally [1]. Theoretically, nonlinear elements are designed on purpose to assist the operation of the machines e.g., the correction of the chromatic aberration and/or slow extraction with the help of sextupole magnets, the enhancement of the Landau damping with the octupole field, etc. On the other hand, the existence of the nonlinear components in the beam line will introduce the nonlinear behavior of the moving particle, and if the driving source of such nonlinear field is strong enough, the particle motion could be so large and lost. In the design stage of the machine components, the error tolerance of the field ought to be specified to assure the expected performance of the machine. In this paper, we employ a turn-by-turn electron position monitoring system to measure the behavior of the perturbed particle at the TLS. The simulation results with the existence of the measured nonlinear field errors are compared with the extracted parameters from the experiments.

\section{TURN-BY-TURN BPM SYSTEM}

We have installed a six-dimensional turn-by-turn phase space monitoring system at the TLS.[2] This system was employed to measure the nonlinear particle motion. The stored beam was perturbed using a horizontal kicker. The two horizontal BPMs are located at the positions where the betatron phase advance differs by $90^{\circ}$ or so. A well-developed on-line data analysis and display window is of help while the experiments are conducted. The lattice working points were set before kicking the stored beam by changing the quadrupole field strength. The coupling in the transverse plane was corrected with skew quadrupoles. Sextupole strengths were adjusted to the desired chromaticities, which were near zero. We focus

cckuo@srrc.gov.tw on the analysis of the third- and fourth-order nonlinear strength of the TLS storage ring.

\section{EXPERIMENTS AND ANALYSIS}

The amplification factor of the measured BPM signal was calibrated using response matrix of the steered beam as well as the bench-measured data. The phase advance of the BPM pair and the phase-space ellipse were obtained by fitting the BPM data and also compared with the on-line machine-model calculations. The measured Poincaré maps are shown in Fig. 2, and 6. The action-angle $(J, \phi)$ of the fitted data is thus given in Fig. 3 , 7, and 9, and the fitted Hamiltonian tori are also displayed. To get the nonlinear betatron de-tuning parameters of the machine, we applied a numerical analysis of the fundamental frequency (NAFF) method to get the betatron tune. Noted that de-coherence effect of the bunched beam should be minimized.

The effective Hamiltonian of particle beam motion in the resonance reference-rotating frame in 1D nonlinear resonance $m v=l$ with small action $I=J$ can be expressed as

$$
\tilde{H}=\delta I+\frac{1}{2} \alpha I^{2}+G_{m, 0, l} I^{m / 2} \cos m \varphi,
$$

where $m, l$ is integer and $v$ is betatron tune.[1] The resonance strength $g=G_{m, 0, l}$, and energy $E=\tilde{H}$ can be fitted provided the proximity of tune $\alpha$ and the de-tuning parameter $\delta$ are measured. The fixed points $\left(I_{f}, \varphi_{f}\right)$ of the Hamiltonian are given by $\sin m \varphi_{f}=0 \quad$ and $\quad \delta+\alpha I_{f} \pm \frac{m}{2} g I_{f}^{m / 2-1}=0$. Hence, the stable and unstable fixed points can be expressed in terms of $\delta, \alpha$ and $g$ for the $m$-order resonance.

\subsection{Third-order}

The ring lattice is a six-fold symmetry triple-bend-achromat lattice with the designed working tune around 7.18 and 4.13 in the horizontal and vertical plane, respectively. The horizontal tune was shifted across the third-order resonance line with $\delta=0.0012$ before kicking the beam horizontally. We got de-tuning $\alpha=-0.0012 \pm 0.0001 \quad(\pi \mathrm{mm} \mathrm{mrad})^{-1}$ and resonance 
strength $g=2.1 \pm 0.5 * 10^{-4}(\pi \mathrm{mm} \mathrm{mrad})^{-1 / 2}$. The island tune becomes $v^{2}{ }_{i s l a n d}=(9 / 2) \sqrt{I_{s f p}}\left(\delta-\alpha I_{s f p}\right)$.

Stable and unstable fixed points can be expressed as $I_{s f p}^{1 / 2}=\left(1.5|g|+\sqrt{(1.5 g)^{2}-4 \alpha \delta}\right) / 2|\alpha| \quad$ and $\left.I_{u f p}^{1 / 2}=|1.5| g \mid-\sqrt{(1.5 g)^{2}-4 \alpha \delta}\right) / 2 \alpha \mid$. The measured island tune $v_{\text {island }}=0.0017 \pm 0.0004$ from FFT of turn-by-turn data as compared with the result from the above equation $v_{\text {island }}=0.0017 \pm 0.0002$. $I_{s f p}=1.29 \pm 0.06$ and $I_{u f p}=0.78 \pm 0.05(\pi \mathrm{mm} \mathrm{mrad})$ from the equations. From the action-angle data in Fig. 3, $I_{s f p}=1.2 \pm 0.1$ and $I_{u f p}=1.0 \pm 0.1(\pi \mathrm{mm} \mathrm{mrad})$.

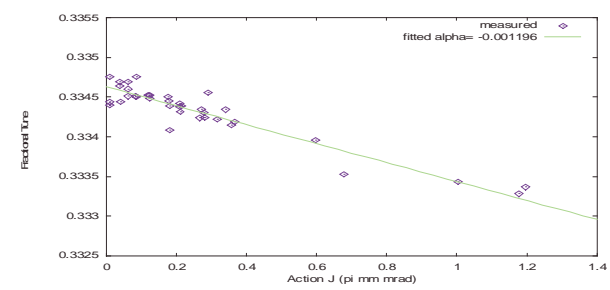

Figure 1: De-tuning parameter around third-order resonance line. $\alpha=-0.0012 \pm 0.0001(\pi \mathrm{mm} \mathrm{mrad})^{-1}$

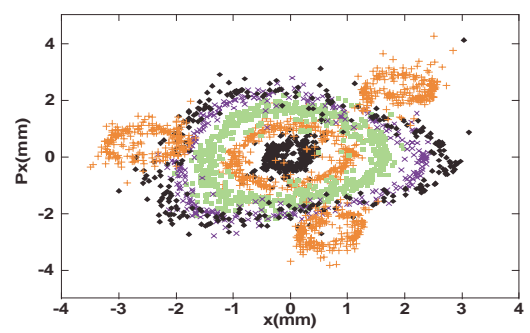

Figure 2: Poincaré map near third-order resonance.

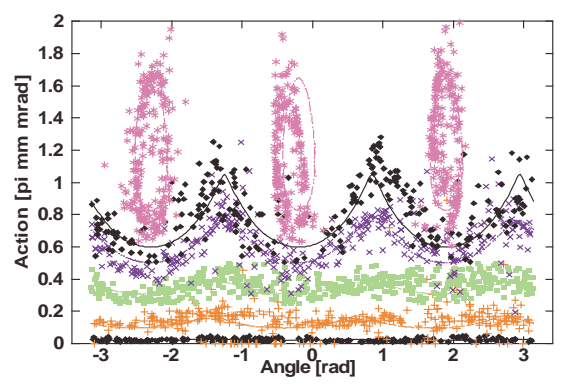

Figure 3: Action-angle near third-order resonance line.

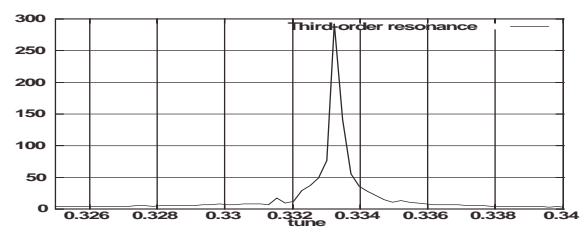

Figure 4: FFT of the third-order resoance. Island tune is shown.

\subsection{Fourth-order}

The horizontal tune was shifted to 7.2538 with $\delta=0.0038$ and measured de-tuning parameter $\alpha=-0.00113 \pm 0.00002(\pi \mathrm{mm} \mathrm{mrad})^{-1}$ as shown in Fig. 5 . In the plot, the action is averaged over the angles. It is found the island width is measurable in the plot. Fitted resonance strength is $g=4.0 \pm 2.0 * 10^{-5}(\pi \mathrm{mm} \mathrm{mrad})^{-1}$. The stable and unstable fixed points as well as the small amplitude island tune for $\alpha<0$ case are given as:

$$
\begin{aligned}
& I_{s f p}=-\frac{\delta}{\alpha+2|g|}, I_{u f p}=\left|-\frac{\delta}{\alpha-2|g|}\right|, \text { and } \\
& v_{\text {island }}=4|\delta| \sqrt{\left|\frac{g}{\alpha+2|g|}\right| .}
\end{aligned}
$$

With $\delta, \alpha$ and $g$ obtained above, we get $I_{s f p}=3.6 \pm 0.1$, $I_{u f p}=3.1 \pm 0.1(\pi \mathrm{mm} \mathrm{mrad})$ and $v_{\text {island }}=0.0030$ $(+0.0007,-0.0010)$. Island tune from the FFT of the experimental data is 0.002 . From Fig.7 of the measured action-angle, $I_{s f p} \approx 3.2(\pi \mathrm{mm} \mathrm{mrad})$.

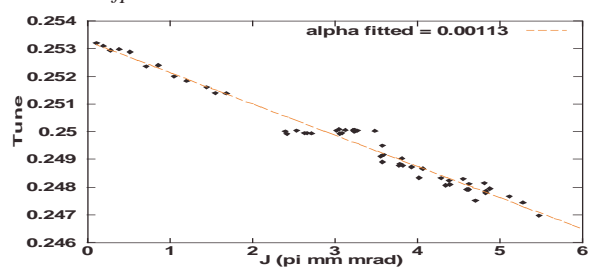

Figure 5: De-tuning parameter around fourth-order resonance line. $\alpha=-0.00113 \pm 0.00002(\pi \mathrm{mm} \mathrm{mrad})^{-1}$

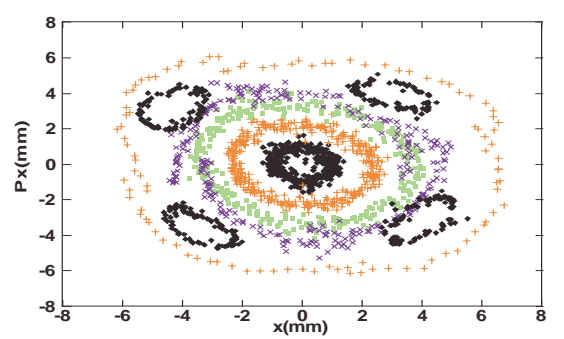

Figure 6: Poincaré map near fourth-order resonance.

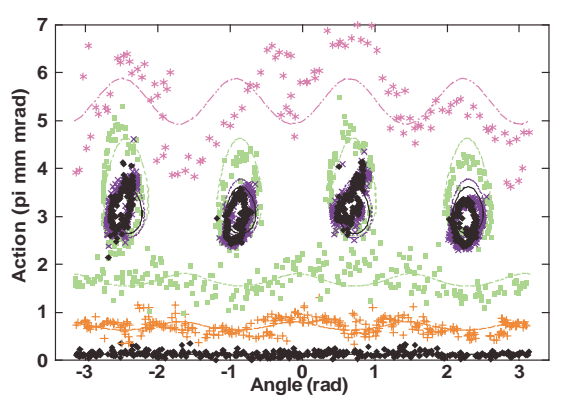

Figure 7: Action-angle near fourth-order resonance line. 


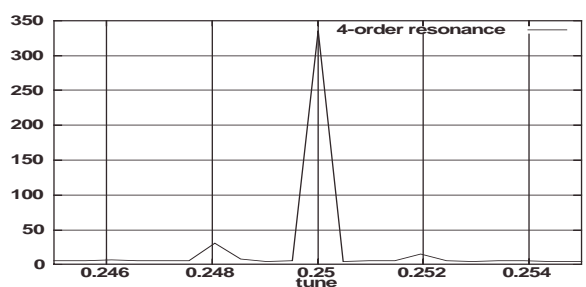

Figure 8: FFT of the fourth-order resonance. Island tune is shown.

\subsection{Fifth-order}

Fifth-order resonance can be also driven by the systematically distributed chromaticity sextupoles as shown in Fig. 9. The initial tune is 7.2095. Measured island tune is 0.011 and $\alpha=-0.00117$.

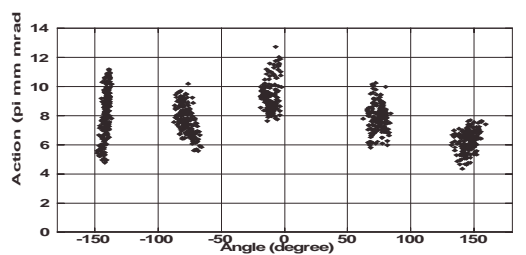

Figure 9: Measured action-angle on fifth-order resonance.

\section{SIMULATION WITH NONLINEAR FIELD ERRORS}

Without nonlinear random errors, the resonance strengths of the third- and fourth-order in the above mentioned conditions will be zero due to the cancellation of the phases in the SRRC lattice. Magnetic field errors were measured in terms of harmonic contents at the operational energy before installing into the ring beam line. Other than skew components, all these errors are put into the simulation code MAD. Due to the lack of the real corresponding error distribution of the magnets, random error distribution along the ring was generated using the measured field statistics. The nonlinear betatron de-tuning parameters contributed from the sextupoles (chromaticity sextupoles and sextupole components of all magnets including insertion devices) near the third-, fourth- and fifth-order resonance are $-0.0012,-0.0016,-0.0018(\pi \mathrm{mm}$ $\mathrm{mrad}^{-1}$, respectively, and are $-0.0011,-0.0014,-0.0017$ for those without errors. Noted that in the simulation, wiggler magnet is turned on. It shows that other higher order nonlinear terms do contribute substantially to the de-tuning effect. It was found that the octupole random errors in quadrupoles needed increase by a factor of 4 to get reasonably reproduced fourth-order resonance strength. This might be due to the inability in reproducing the exact random octupole field distribution in the simulation or the octupole components in quadrupoles were under-estimated. The de-tuning parameters, the resonance strengths, the island tunes are listed in Table 1.

\section{CONCLUSION}

In this study, we found that the non-linear beam dynamics in the transverse plan can be measured and compared with the simulation results. The measured data in third-order and fourth- as well as fifth-order resonance lines are reasonably reproduced using MAD simulation code, in which measured field errors are included.

\section{ACKNOWLEDGEMENT}

The authors thank the help and discussion from M.H. Wang, G.H. Luo, and especially from S.Y. Lee and A. Chao.

Table 1: Simulated parameters with error inputs.

\begin{tabular}{|c|l|l|}
\hline & Third-order & Fourth-order \\
\hline$\alpha$ & -0.00088 & -0.00128 \\
\hline$g$ & $6 \mathrm{e}-4$ & $7 \mathrm{e}-5$ \\
\hline$v_{\text {island }}$ & 0.0013 & 0.002 \\
\hline
\end{tabular}

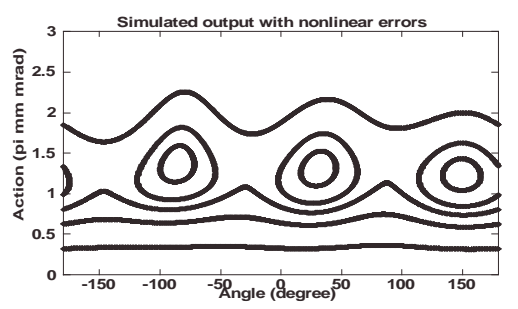

Figure 10: Third-order resonance tracking in the horizontal plan with measured field errors using MAD program. Initial tune is 7.3344 .

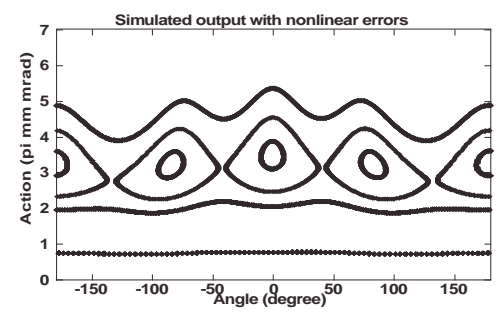

Figure 11: Same set of field errors as in Fig. 10 for the fourth-order resonance map. Initial tune is 7.2538.

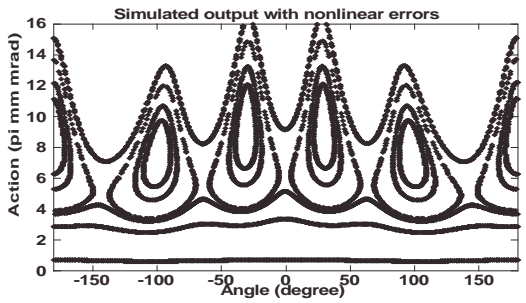

Figure 12: Simulated action-angle near fifth-order resonance. Initial tune $=7.2095$.

\section{REFERENCES}

[1] R. Ruth, AIP Conf. Proc. No. 153 (1984) p. 150

A. Chao et al., Phys. Rev. Lett. 61, 2752 (1988)

S.Y. Lee et al., Phys. Rev. Lett. 67, 3768 (1991)

Y. Wang et al., Phys. Rev. E 49, 5697 (1994)

[2] J. Chen et al., 2412, PAC99 (1999) 